\title{
Nuclear to non-nuclear Pmel17/gp100 expression (HMB45 staining) as a discriminator between benign and malignant melanocytic lesions
}

\author{
Bonnie E Gould Rothberg ${ }^{1}$, Christopher B Moeder ${ }^{1}$, Harriet Kluger ${ }^{2}$, Ruth Halaban ${ }^{3}$,
} David E Elder ${ }^{4}$, George F Murphy ${ }^{5}$, Alexander Lazar ${ }^{6}$, Victor Prieto ${ }^{6}$, Lynn McDivitt Duncan $^{7}$ and David L Rimm ${ }^{1}$

${ }^{1}$ Department of Pathology, Yale University School of Medicine, New Haven, CT, USA; ${ }^{2}$ Department of Medicine, Yale University School of Medicine, New Haven, CT, USA; ${ }^{3}$ Department of Dermatology, Yale University School of Medicine, New Haven, CT, USA; ${ }^{4}$ Department of Pathology, University of Pennsylvania, Philadelphia, PA, USA; ${ }^{5}$ Department of Pathology, Brigham and Women's Hospital, Boston, MA, USA; ${ }^{6}$ Department of Pathology, MD Anderson Cancer Center, Houston, TX, USA and ${ }^{7}$ Department of Pathology, Massachusetts General Hospital, Boston, MA, USA

\begin{abstract}
HMB45 is a mouse monoclonal antibody raised against Pmel17/gp100, a melanoma-specific marker, which is routinely used in the diagnosis of primary cutaneous malignant melanoma. The standard expression pattern for a positive HMB45 staining result on immunohistochemistry is based upon the results of chromogenic-based methods. We re-evaluated the patterns of HMB45 staining across the 480-core 'SPORE melanoma progression array' containing lesions representing the spectrum of melanocytic lesions ranging from thin nevus to visceral metastasis using the fluorescence-based staining technique and automated quantitative analysis (AQUA) of the obtained digital images. The methods validated the expected cytoplasmic HMB45 staining pattern in $70 / 108$ malignant lesions and in the epithelial components of nevus specimens. However, the fluorescence-based approach revealed a nuclear gp100 localization present in the dermal component of all nevi that was not seen before. This nuclear localization could not be observed on routine chromogenic stains, because the standard hematoxylin nuclear counterstain overwhelms the weak nuclear HMB45 stain. The thin $(0.450 \pm 0.253)$ and thick $(0.513 \pm 0.227)$ nevi had strongly positive mean In(nuclear/non-nuclear AQUA score ratios), which are significantly higher than those from the group of malignant lesions $(P<0.0001)$. This finding was reproduced on a smaller but independent progression array composed of nevi and melanomas from the Yale Pathology archives $(P<0.01)$. The odds ratio associated with a sample being a nevus was $2.24(95 \% \mathrm{Cl}$ : $1.87-2.69$, $\boldsymbol{P}<0.0001$ ) for each 0.1 unit increase of the In(nuclear/non-nuclear AQUA score ratio) to yield an ROC curve with 0.93 units of area and a simultaneously maximized sensitivity of 0.92 and specificity of 0.80 for distinguishing benign nevi from malignant melanomas. On the basis of this preliminary study, we propose that the ratio of nuclear to non-nuclear HMB45 staining may be useful for diagnostic challenges in melanocytic lesions. Modern Pathology (2008) 21, 1121-1129; doi:10.1038/modpathol.2008.100; published online 13 June 2008
\end{abstract}

Keywords: immunohistochemistry; immunofluorescence; nevus; melanoma; diagnostic; cutaneous

The human homolog of the mouse silver protein (gp100 or Pmel17) is a melanocyte-specific type I membrane protein required for proper formation of melanosomal fibrils, which facilitates the maturation of stage I premelanosomes to stage II. ${ }^{1,2} \mathrm{Gp} 100$

Correspondence: Dr DL Rimm, MD, PhD, Department of Pathology, Yale University School of Medicine, 310 Cedar St, PO Box 208023, New Haven, CT 06520-8023, USA.

E-mail: david.rimm@yale.edu

Received 18 February 2008; revised 01 May 2008; accepted 04 May 2008; published online 13 June 2008 has long been used as a melanocyte/melanosome marker in the diagnosis of primary cutaneous melanoma and in the identification of melanoma cells in sentinel lymph node biopsies.,4 The recognition of gp100 is commonly performed with HMB45, a mouse monoclonal antibody that specifically reacts with the glycosylated form of gp100 restricted to the fibrillar matrix of stage II premelanosomes. ${ }^{5-7}$

The distribution of HMB45 staining patterns for benign and malignant melanocytic lesions has been characterized by multiple groups using routine 
techniques that employ 3-3'-diaminobenzidine (DAB) or $3^{\prime}$-aminoethyl-carbazole (AEC) as a chromogen. HMB45 staining is observed in over $95 \%$ of epithelioid cutaneous melanomas with most lesions yielding over $50 \%$ positive staining of the cytoplasmic portion of the cells. ${ }^{8-11}$ Spindle cell and desmoplastic melanomas, however, tend to be HMB45-negative under standard antigen retrieval and immunostaining techniques. ${ }^{12}$ Aggressive antigen retrieval can induce HMB45 staining in spindle cell but not in desmoplastic melanomas. ${ }^{12,13}$ Evaluation of a series of metastatic lesions from 121 individuals with stage IV disease revealed substantial heterogeneity in HMB45 staining patterns with no staining in $26(21.5 \%)$ of the lesions and onethird of the remaining lesions demonstrating either weak, moderate or strong staining. ${ }^{14}$

Analysis of HMB45 staining patterns in benign melanocytic lesions is stratified by the histologic type of lesion and location of the specific nevoid cells relative to the dermal-epidermal junction. Compound melanocytic nevi are characterized by cytoplasmic HMB45 reactivity within the epidermal component of the lesion, focal positivity in the junctional regions and subsequent loss of stain in

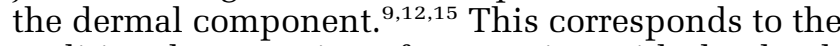
traditional conception of maturation with the depth of melanocytes in nevi. Ordinary dermal nevi are HMB45 negative, ${ }^{16}$ whereas dysplastic nevi ${ }^{17}$ and blue nevi ${ }^{18,19}$ are positive.

Chromogenic stains, however, suffer from several drawbacks including a limited dynamic range, lack of robust standardization methods and the reliance mostly on the subjective interpretation of a human observer to classify staining patterns into discrete ordinal categories, although automated readers have been developed..$^{20}$ This limitation was addressed by the optimization of fluorescence-based immunohistochemistry techniques on tissue microarrays coupled with the development of automated methods of quantification of staining intensity as a continuous parameter. Here, we re-evaluate the patterns of HMB45 staining across a tissue microarray containing a series of 167 unique melanocytic lesions ranging from thin benign nevi to visceral melanoma metastases using fluorescence-based immunohistochemistry followed by automated quantitative analysis (AQUA) of protein expression. ${ }^{21}$ This technique revealed, for the first time, the patterns of nuclear gp100 expression in benign nevi suggesting its potential diagnostic utility to determine the ratio of nuclear to non-nuclear HMB45 staining for discriminating benign from malignant melanocytic lesions.

\section{Materials and methods}

\section{Sample Selection}

Two separate collections of melanoma samples were used for this analysis. The SPORE progression array was constructed by the Harvard tissue microarray facility using the standard method ${ }^{22}$ and included a series of randomly-selected, formalin-fixed, paraffin-embedded melanocytic lesions retrieved from the archives at Harvard University, the University of Pennsylvania and the MD Anderson Cancer Center. The array includes 21 thin $(<1 \mathrm{~mm})$ nevi, 15 thick ( $>1 \mathrm{~mm}$ ) nevi, 38 radial growth phase $(<1 \mathrm{~mm})$ primary melanomas, 20 vertical growth phase ( $>1 \mathrm{~mm}$ ) primary melanomas, 28 lymph node metastases and 45 metastases to visceral organs. Duplicate $0.6 \mathrm{~mm}$ cores were obtained from each thin lesion as well as from the set of metastatic lesions. For the thick lesions, three depths of invasion relative to the skin edge were defined (superficial, mid-level and deep) and duplicate $0.6 \mathrm{~mm}$ cores were taken at each of these depths to produce a total of six cores from each tumor.

The Yale Melanoma Boutique array was constructed by the Yale tissue microarray facility using formalin-fixed, paraffin-embedded archival material. It included single $0.6 \mathrm{~mm}$ cores from 20 benign nevi, 20 vertical growth phase primary melanomas and 20 metastases, the latter representing lesions from subcutaneous, lymph node and visceral sites. In addition, the tissue microarray included as controls $0.6 \mathrm{~mm}$ cores from paraffin-embedded pellets of 16 melanoma and 18 nonmelanoma cell lines grown in culture to confluency and harvested into $10 \%$ neutral-buffered formalin.

\section{Immunohistochemistry}

Five micron sections were cut from each array master block, adhered to the recipient glass slide using an ultraviolet cross-linkable tape transfer system (Instrumedics Inc., Hackensack, NJ), dipped in paraffin and stored in a nitrogen chamber until use. Slides were deparaffinized using two xylene exchanges followed by rehydration in ethanol gradient. Antigen retrieval was performed by boiling the slides in buffer containing $6.5 \mathrm{mM}$ sodium citrate $(\mathrm{pH}=6.0)$ in a sealed pressure cooker for $10 \mathrm{~min}$. The slides were immersed in absolute methanol containing $0.75 \%$ hydrogen peroxide for $30 \mathrm{~min}$ to neutralize endogenous peroxidase activity, followed by a $30 \mathrm{~min}$ incubation in $0.3 \%$ bovine serum albumin (BSA) dissolved in $1 \mathrm{M}$ tris-buffered saline (TBS; $\mathrm{pH}=8.0$ ) to block nonspecific binding.

Fluorescence-based immunohistochemical staining was performed by multiplexing the prediluted HMB45 mAb (BioGenex, San Ramon, CA) with rabbit polyclonal anti-S100B (Dako Cytomation, Carpenteria, CA) at 1:200 dilution. The latter was used to define the 'tumor mask,' which discriminates the regions corresponding to melanoma from the surrounding tissue within the histospot in the absence of simultaneous H\&E staining. Primary antibodies were incubated at $4^{\circ} \mathrm{C}$ overnight. Visualization was accomplished using fluorescent probes. The secondary fluorescent antibodies, 
Alexa-546-conjugated goat antirabbit (1:100 dilution, Molecular Probes, Eugene, OR) diluted into Envision antimouse (neat; DAKO) were applied for $1 \mathrm{~h}$ at room temperature. The slides were washed three times for 10 min with $1 \mathrm{M}$ TBS, incubated with cyanine-5-conjugated tyramide (Perkin-Elmer, Wellesley, MA) for $10 \mathrm{~min}$, and then with $0.01 \mathrm{mg} / \mathrm{ml}$ 4',6-diamidino-2-phenylindole (DAPI) for 20-min for nuclear compartment visualization. The slides were mounted with Prolong-Gold antifade reagent (Invitrogen), sealed with a nylon-based lacquer and stored in the dark until scoring.

For brown-stain-based immunohistochemistry, the prediluted HMB45 antibody was applied neat for $1 \mathrm{~h}$ at room temperature and visualized using Envision $^{\mathrm{TM}}$ with a 3-3'-diaminobenzidine chromogen. Counterstaining was achieved using Meyer's hematoxylin. Evaluation of chromogen-stained arrays and conventional imaging was performed by a single pathologist (DLR).

\section{Digital Image Capture and Automated Quantitative Analysis (AQUA ${ }^{\circledR}$ ) of Protein Expression}

The AQUA image acquisition and analysis was performed as described previously. ${ }^{21}$ Briefly, stained slides were imaged on a modified computer-controlled epifluorescence microscope (Olympus BX-51 with xy-stage and $\mathrm{z}$ controller) illuminated by a high-pressure mercury bulb (Photonic Solutions, Missisauga, ON) with a high-resolution monochromatic camera (Cooke Corporation, Romulus, MI). Following user optimization of the focus, sets of monochromatic, high-resolution $(1024 \times 1024$, $0.5 \mu \mathrm{m}$ ) images were captured for each histospot, for each of the DAPI, Alexa-546 and Cy5 fluorescent channels. Two images were captured for each channel: one in the plane of focus and the other $8 \mu \mathrm{m}$ below it. The gp100 staining patterns were captured by a $65 \mathrm{~ms}$ exposure time in the cyanine- 5 channel on the SPORE progression array and $57 \mathrm{~ms}$ for the Yale Melanoma Boutique array. The Alexa546 signal representing S100B staining was binary gated to indicate whether a pixel is within the tumor mask ('on') or not ('off'). Further subcellular compartmentalization defined nuclear from non-nuclear regions within the tumor. The nuclear compartment was defined as the subset of pixels that demonstrated any DAPI staining within the plane of focus. This was required to compensate for the 3-dimensional thickness of the tumor sections, which can blur the discrimination of the nuclear boundary. The non-nuclear compartment was then defined as all pixels assigned to the tumor mask but are not included within the nuclear compartment. Finally, gp100 expression levels were determined in an automated fashion, blinded to any a priori clinical information, from the images acquired under the Cy5 channel to obtain a relative pixel intensity restricted to the signal emanating from the plane of focus. The final AQUA score for the entire tumor mask or any of its subcellular compartments was calculated as the average AQUA score for each of the individual pixels included in the selected compartment and was reported on a scale of $0-255$.

\section{Statistical Analysis}

Cores whose tumor mask covered $<5 \%$ of the total histospot area were dropped from further analysis. The raw AQUA scores for the total area under the tumor mask as well as each of the specific nuclear and non-nuclear compartments were used. To compare the intensity of nuclear to non-nuclear staining, the natural log of the ratio of nuclear to non-nuclear AQUA scores was calculated.

Evaluation of the AQUA scores associated with HMB45 staining in each of the three defined compartments and the ratio of nuclear to nonnuclear staining across the progression of melanocytic lesions was performed by the analysis of variance (ANOVA). To account for the redundant cores within the SPORE progression array, a mixedeffect ANOVA model was used which included a random effects parameter for sample. Testing for the significance of individual pairwise comparisons in each ANOVA was adjusted using the Tukey method for multiple comparisons. Assessment of a linear trend across lesion progression was accomplished by mixed model linear regression incorporating a random effects parameter for sample following ordinalization of the lesion type. Calculation of the receiver-operator characteristic curve was performed from the logistic regression model measuring the likelihood that a given sample is a benign nevus. For all analyses, significant associations were defined as $P<0.05$. All statistical analyses were performed using SAS version 9.1.3 and Statview (SAS Institute, Cary, NC).

\section{Results}

\section{HMB45 Staining Trends Across Core Depth in Thick Lesions}

AQUA was performed on the SPORE array with 334 histospots representing 136 samples available for analysis. To determine the trend of gp100 expression across core depth among the thick nevi, the distributions of AQUA scores for superficial, mid-level and deep cores from each thick nevus were compared. No significant difference in the distribution of AQUA was observed among the three tissue depths for either the nuclear $(P=0.42)$, the non-nuclear compartments $(P=0.75)$, or the aggregate AQUA score when the entire area under the tumor mask was considered $(P=0.76$; Figure 1a-b). 

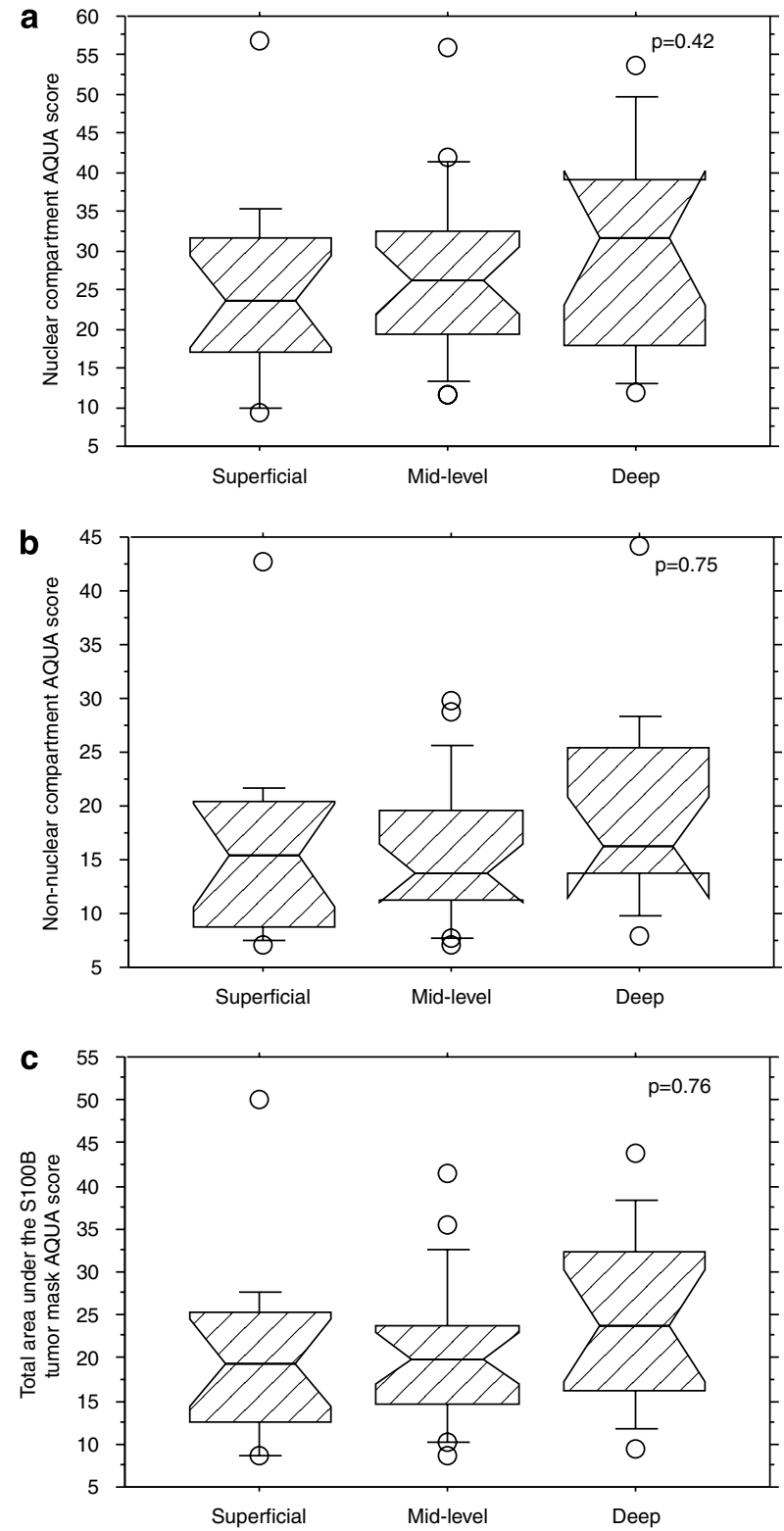

Figure 1 Box plots showing the relative distributions of AQUA scores for (a) nuclear compartment, (b) non-nuclear compartment and (c) for total area under the tumor mask for the superficial, mid-level and deep regions of the thick nevi (as indicated). No significant differences were observed using mixed model ANOVA $(P>0.20)$.

\section{gp100 Expression Across Melanocytic Lesion Progression}

Representative HMB45 staining patterns for each class of lesion spotted on the tissue microarray are shown in Figure 2a-d. Among the specimens from primary and metastatic lesions, HMB45 most strongly stained the cytoplasmic regions (nonnuclear compartment) of the cells although some degree of nuclear staining could be observed. Setting an AQUA score of 20 as the maximal cutoff level designating moderate to strong HMB45 staining in the non-nuclear compartment, cytoplasmic staining was observed in 16/25 radial growth phase primaries, 14/19 vertical growth phase primaries, 16/24 lymph node metastases and $24 / 40$ visceral metastases. Cytoplasmic staining was present in none of the thin nevi and only in one thick nevus. Interestingly, definitive nuclear staining was observed in the dermal component of all thin and thick nevi, whereas only three metastatic melanoma lesions demonstrated any nuclear staining and weak cytoplasmic HMB45 staining. Focal staining was observed in two of these cases, with the third lesion displaying a punctuated nuclear staining pattern throughout the histospot.

Examination HMB45 reactivity of nevi using conventional immunohistochemistry with a DAB chromogen and hematoxylin counterstain of the Yale Melanoma Boutique array shows no appreciable staining (Figure 3a). We hypothesize that the low levels of expression are obscured by the hematoxylin counterstain. To verify that the fluorescence-based staining described above (Figure 2) was not an artifact of the visualization method, a second, serial section of the Yale Melanoma Boutique array was stained using DAB as the chromogen but without the hematoxylin counterstain. Faint DAB stain was observed in the nuclei of nevoid melanocytes in the absence of hematoxylin (Figure $3 \mathrm{~b})$, but the intensity of the nuclear counterstain overwhelms the faint HMB45-produced DAB stain (Figure 3a). Figure 3c and d show a more typical example of HMB-45 staining of a malignant melanoma with strong cytoplasmic staining. The absence of counterstain in this case (Figure 3d) does not reveal the pale nuclear staining seen in the nevus in Figure 3b.

\section{The Ratio of Nuclear/Non-Nuclear AQUA Scores is a Significant Discriminator of Benign Melanocytic Lesions}

To extend our observation defining a distinct, predominantly nuclear HMB45 staining pattern for benign melanocytic lesions that is significantly different from the predominantly cytoplasmic distribution seen in the malignant lesions, the ratio of nuclear to non-nuclear AQUA scores was evaluated as a possible discriminator of benign melanocytic lesions. To normalize the distribution of ratios, the natural log transformation of the ratio was selected as the preferred parameter. Using linear mixed models, both the thin $(0.450 \pm 0.253)$ and thick $(0.513 \pm 0.227)$ nevi had strongly positive mean ln(nuclear/non-nuclear ratios) that were significantly different $(P<0.0001)$ from the group of malignant lesions whose means \pm s.d. range from $0.128 \pm 0.285$ to $0.055 \pm 0.186$ (Figure $4 \mathrm{a}$ ). To determine the reproducibility of this finding on an independent collection of benign and malignant melanocytic lesions, the Yale Melanoma Boutique array was stained with the HMB45 antibody using 
a

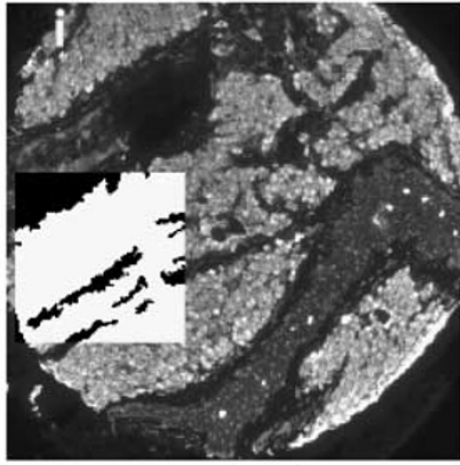

b

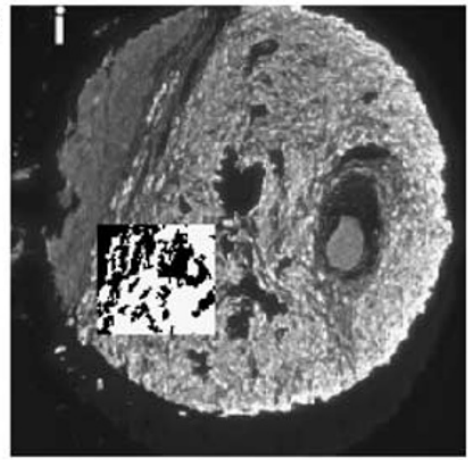

c

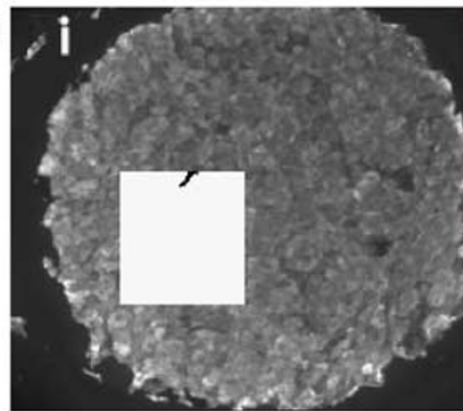

d

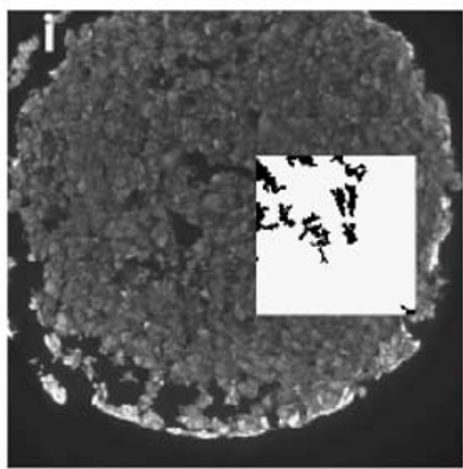

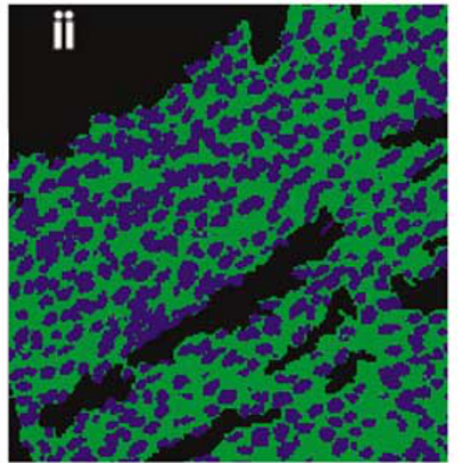
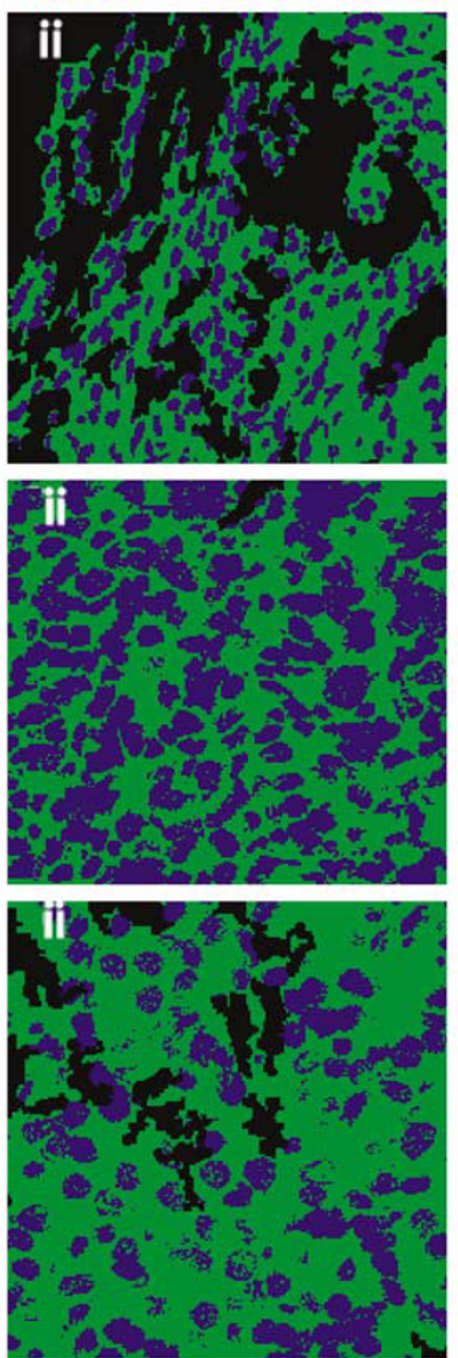
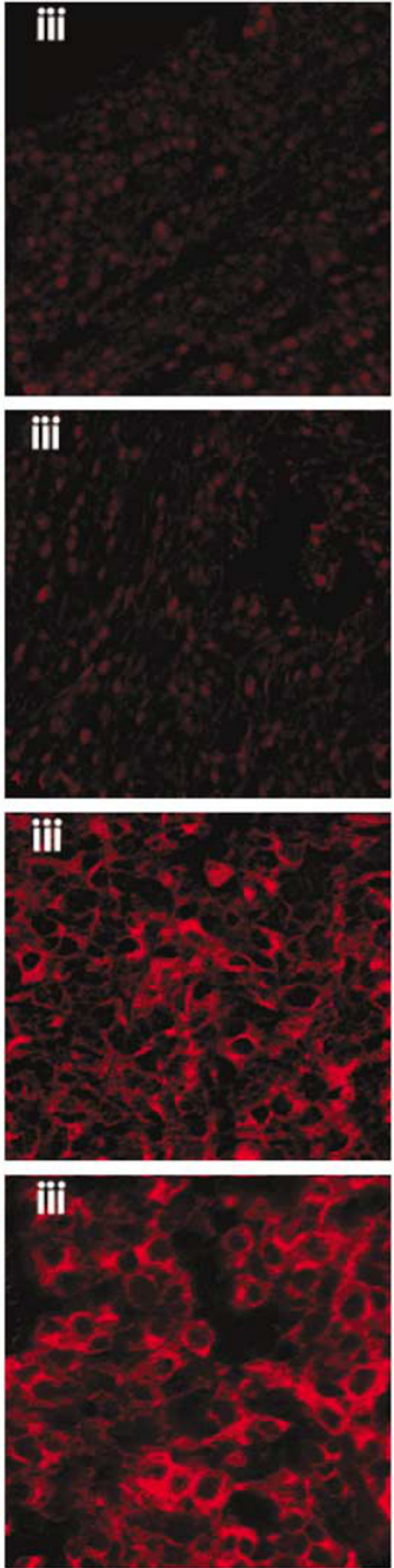

Figure 2 Representative images of a thin nevus (a), thick nevus at mid-level (b), a thick primary (c) and a visceral metastasis (d). For each image series, the first image captures S100B staining in the Alexa-546 channel and the remaining two images represent a magnification of the inset region. The solid inset represents the conversion of S100B staining into the binary-gated tumor mask, which differentiates cells from the melanocytic lesion from surrounding stroma and matrix. The composite of the DAPI stain-positive nuclear (blue) and DAPI stain-negative non-nuclear (green) compartments within the tumor mask (aii-dii), and the distribution of HMB45 within these compartments (images aiii-diii) is presented. In both thin and thick nevi, HMB45 is concentrated in the nuclear compartment, whereas in the malignant lesions, HMB45 distribution is predominantly cytoplasmic.

fluorescence-based methods. Twenty benign nevi, 20 vertical growth phase primaries and 18 metastases yielded histospots with sufficient material for analysis. The mean $\ln ($ nuclear/non-nuclear ratio) for benign nevi of $0.68 \pm 0.22$ was significantly higher than those from vertical growth phase primaries $(0.16 \pm 0.32 ; P<0.0001)$ and metastases $(0.42 \pm 0.37$; $P=0.01$; Figure 4b). 

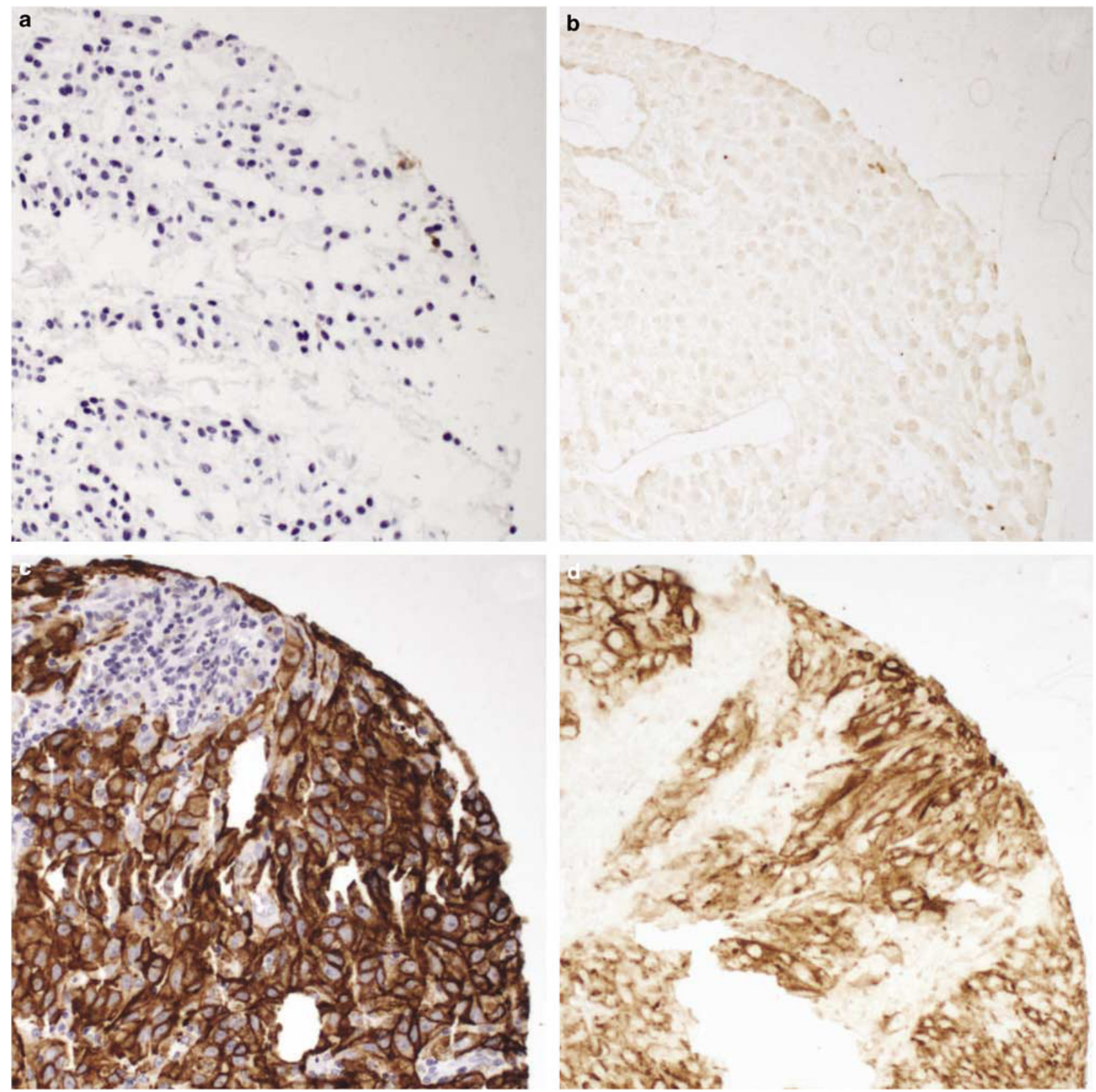

Figure 3 HMB45 staining of melanocytic lesions using conventional immunohistochemistry with a DAB chromogen. In the presence of hematoxylin counterstain, a nevus from the Yale Melanoma Boutique collection (a) shows no appreciable HMB45 staining. In the absence of counterstain, faint nuclear DAB stain can be observed in a serial section (b). A representative primary melanoma lesion from the same collection shows intense cytoplasmic staining that can be visualized in the presence (c) or absence (d) of hematoxylin counterstain, but no nuclear reactivity is seen, even in the absence of counterstain.

To evaluate the predictive capability of the $\ln ($ nuclear/non-nuclear ratio), logistic regression was executed to determine the likelihood of a sample being a nevus, yielding an odds ratio of $2.24 \quad(95 \%$ CI: $1.87-2.69 ; \quad P<0.0001)$ for each 0.1 unit increase in $\ln$ (nuclear/nonnuclear ratio). A receiver-operator characteristic curve was constructed from these data across all possible dichotomizing cut points covering the full range of the $\ln$ (nuclear/non-nuclear ratio) scores, which produced 0.93 units of area and, at the shoulder of the curve, created by designating an $\ln$ (nuclear/non-nuclear HMB45 AQUA score ratio) $>0.154$ as positively identifying a nevus, a maximized simultaneous sensitivity of 0.92 and specificity of 0.80 for discriminating benign nevi from malignant melanocytic lesions (Figure 5). 

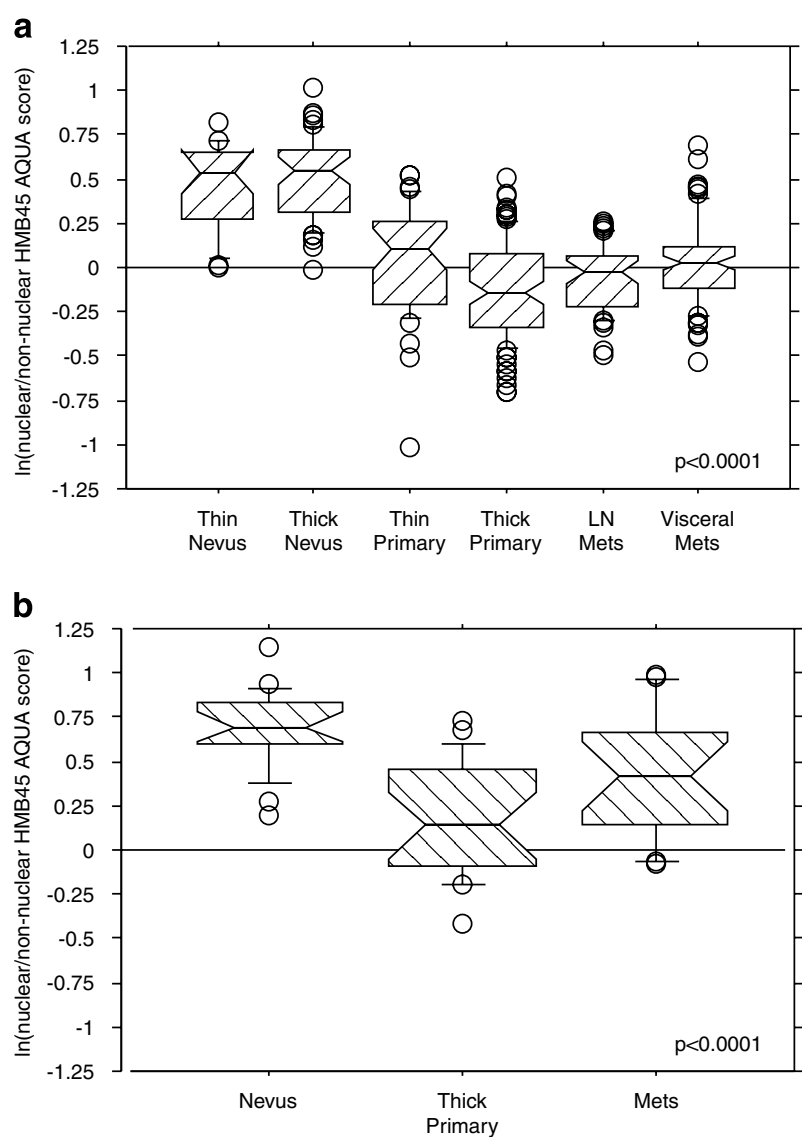

Figure 4 (a) Box plot describing the distributions of ln(nuclear/ non-nuclear compartment AQUA scores) for the six levels of disease spotted on the melanoma SPORE progression array. Thin and thick nevi display elevated ratios that are significantly different from each category of malignant lesions (mixed model ANOVA pairwise comparison $P$-values all $<0.0001$ ). (b) Box plot describing the distribution of $\ln$ (nuclear/non-nuclear compartment AQUA scores) for nevi, thick primaries and metastatic lesions in the Yale Boutique Array (fixed effects ANOVA pairwise comparison $P$-values all $<0.01)$.

\section{Discussion}

HMB45 reactivity is a cornerstone for the pathologic identification of melanocytic lesions. The cytoplasm of epithelioid cutaneous melanomas characteristically stains positively for HMB45, a diagnostic feature frequently used when pathologists evaluate malignancies of unknown primary. ${ }^{4}$ Since HMB45 was first described in $1986,{ }^{23}$ the body of literature on the patterns of HMB45 staining among distinct classes of melanocytic lesions has been largely derived from nonfluorescent-based immunohistochemical techniques with brown or red colored chromogens. As such, their results are limited by the usual issues associated with any chromogen evaluation, including lack of dynamic range, lack of standardization, the reliance on subjective scoring methods and the categorization of scores into discrete categories. In this analysis, we reconsidered patterns of HMB45 reactivity through the application of immunofluorescence-based staining techniques.

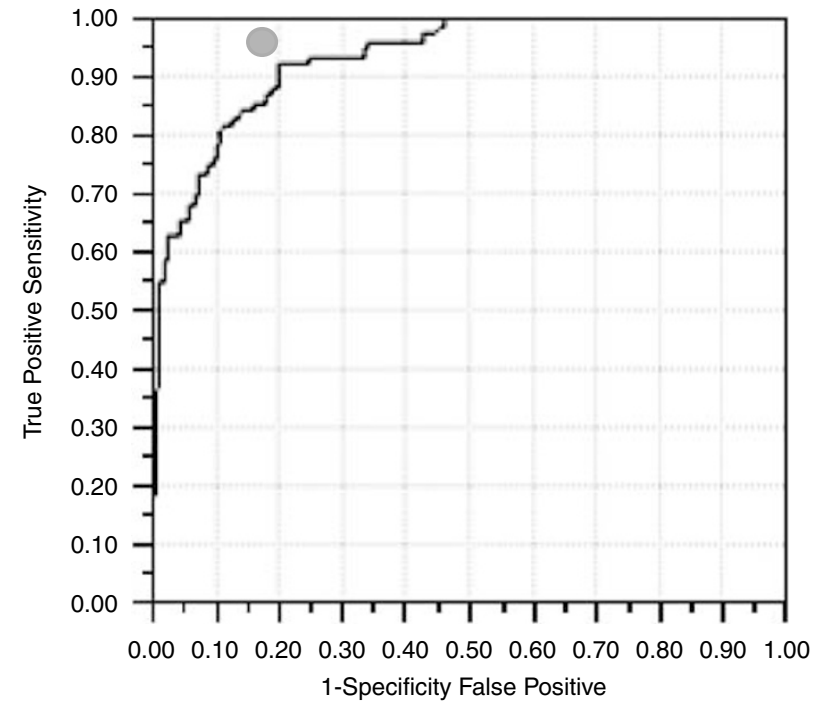

Figure 5 Receiver-operator characteristic curve describing the predictive capabilities of the $\ln$ (nuclear/non-nuclear compartment AQUA score ratio) for distinguishing a nevus from a malignant lesion. The shoulder of the curve (green dot) identifies the simultaneously maximized sensitivity $=0.92$ and specificity $=0.80$.

The increased sensitivity, combined with the lack of obscuring counterstains, revealed an interesting expression patterns that have not been previously described for this protein.

Several reports in the literature documented an inverse trend of gp100 expression in compound nevi as the lesion extends from epithelial to dermal surroundings. ${ }^{9,12,15}$ In our analysis of 15 compound nevi, we did not detect a significant aggregate difference in the non-nuclear AQUA score across serial cores representing superficial, mid-level and deep regions of each lesion. In the light of this result, review of the S100 staining patterns among the set of superficial lesions revealed only one core with a clearly identifiable epidermal component of the nevus, which did demonstrate strong cytoplasmic HMB45 staining that was absent in the adjacent dermal component. Mid-level and deep sections from this same specimen did not reveal any cytoplasmic HMB45 staining. Interestingly, two other thick nevi had evidence of cytoplasmic staining in epithelial regions captured in their mid-level sections. As no previous assessment of HMB45 staining in compound nevi used either tissue microarray technology or immunofluorescent methods, a more targeted study designed to capture epidermal elements of compound nevi is needed to verify this result using AQUA methodology.

Our analysis, however, did reveal a previously undocumented finding of nuclear staining of dermal nevus cells with the HMB45 antibody in the absence of cytoplasmic staining. Interestingly, while faint HMB45 nuclear staining can be observed in nevi using conventional nonfluorescent chromogen stain methods in the absence of a nuclear counterstain, 
the application of standard nuclear stains such as Meyer's hematoxylin overwhelms the HMB45 staining. This observation highlights a specific niche for fluorescence-based immunohistochemical techniques where the detection of antigens expressed at low levels can be optimized by both leveraging the increased dynamic range associated with fluorescent staining compared with conventional brown stain methods ${ }^{20}$ and using image capture techniques that allow for extended exposure times to capture light signals below the detection capabilities of the human eye. Our observation also highlights an underappreciated weakness of conventional techniques where application of the counterstain can hinder target visualization.

By using the $\ln$ (nuclear/non-nuclear AQUA score ratio), which maximizes the contrast between the nuclear staining pattern observed among the benign nevi and the predominantly cytoplasmic staining pattern observed in the malignant lesions, we were able to demonstrate an odds ratio of 2.24 for being a nevus vs a malignant lesion with each increase of 0.1 in the measured ratio. This logistic association was converted into a receiver-operator characteristic curve that demonstrated an area of 0.93 and simultaneously maximized sensitivity of 0.92 and specificity of 0.80 , suggesting the potential utility of this ratio as an additional diagnostic tool in the characterization of melanocytic lesions. However, these cases were all selected from various institutions as classic examples of the histotype. The selective value of the assay and the true sensitivity and specificity of the test remains to be determined in tests of a wider range of serially collected melanocytic lesions.

One diagnostic dilemma that may benefit from this assay is the ability to distinguish a Spitz nevus from Spitzoid melanoma. Currently, no definitive criteria exist that can triage among the two types of lesions and a misdiagnosis can increase patient morbidity and mortality through either undertreatment of a melanoma or overtreatment of a benign lesion with unnecessary surgery and other systemic therapies in addition to the burden of psychological stress associated with the misdiagnosis and its sequelae. ${ }^{24}$ Of greater concern is the estimate from one group that, among children, more than $40 \%$ of cases diagnosed as Spitz nevus are actually a malignancy that has been misdiagnosed. ${ }^{25}$ Few studies have been published characterizing the differential expression of any marker between Spitz nevi and spitzoid melanomas with only CD99, a transmembrane protein associated with the epithelial-mesenchymal transformation, showing moderate discriminant activity between the two lesions. ${ }^{26}$ Two immunohistochemical studies of HMB45 staining in Spitz nevi have been published, one reporting on 15 cases, the other on 30 . Both studies describe heterogeneous staining patterns where half of the lesions were positive for HMB45 in the dermal cells and the remaining lesions divided between no staining and staining only in the epithelial component. ${ }^{8,10}$ None of these studies commented on longterm follow-up of these cases. One report evaluating a small series of spitzoid melanomas suggested that these lesions may have more consistent dermal cytoplasmic HMB45 staining compared to ordinary benign nevi. ${ }^{27} \mathrm{~A}$ targeted follow-up study testing the ability of HMB45 to discriminate benign and malignant Spitzoid lesions is planned.

\section{Acknowledgement}

This work was funded by NIH RO-1 CA 114277 to Dr Rimm and Yale SPORE in Skin Cancer Grants 1P50 CA121974 to Dr R Halaban.

\section{Disclosure/conflict of interest}

David L Rimm is a stock holder, scientific founder and consultant to HistoRx, a private corporation to which Yale University has given exclusive right to produce and distribute the software and technologies embedded in AQUA. Yale University retains patent rights for the AQUA technology.

\section{References}

1 Hoashi T, Muller J, Vieira WD, et al. The repeat domain of the melanosomal matrix protein PMEL17/GP100 is required for the formation of organellar fibers. J Biol Chem 2006;281:21198-21208.

2 Valencia JC, Hoashi T, Pawelek JM, et al. Pmel17: controversial indeed but critical to melanocyte function. Pigment Cell Res 2006;19:250-252; author reply $3-7$.

3 Satzger I, Volker B, Meier A, et al. Prognostic significance of isolated HMB45 or Melan A positive cells in Melanoma sentinel lymph nodes. Am J Surg Pathol 2007;31:1175-1180.

4 Yaziji H, Gown AM. Immunohistochemical markers of melanocytic tumors. Int J Surg Pathol 2003;11:11-15.

5 Berson JF, Harper DC, Tenza D, et al. Pmel17 initiates premelanosome morphogenesis within multivesicular bodies. Mol Biol Cell 2001;12:3451-3464.

6 Kushimoto T, Basrur V, Valencia J, et al. A model for melanosome biogenesis based on the purification and analysis of early melanosomes. Proc Natl Acad Sci USA 2001;98:10698-10703.

7 Raposo G, Tenza D, Murphy DM, et al. Distinct protein sorting and localization to premelanosomes, melanosomes, and lysosomes in pigmented melanocytic cells. J Cell Biol 2001;152:809-824.

8 Bergman R, Dromi R, Trau $\mathrm{H}$, et al. The pattern of HMB-45 antibody staining in compound Spitz nevi. Am J Dermatopathol 1995;17:542-546.

9 de Vries TJ, Fourkour A, Wobbes T, et al. Heterogeneous expression of immunotherapy candidate proteins gp100, MART-1, and tyrosinase in human melanoma cell lines and in human melanocytic lesions. Cancer Res 1997;57:3223-3229. 
10 Lazzaro B, Elder DE, Rebers A, et al. Immunophenotyping of compound and spitz nevi and vertical growth-phase melanomas using a panel of monoclonal antibodies reactive in paraffin sections. J Invest Dermatol 1993;100:313S-317S.

11 Niezabitowski A, Czajecki K, Rys J, et al. Prognostic evaluation of cutaneous malignant melanoma: a clinicopathologic and immunohistochemical study. J Surg Oncol 1999;70:150-160.

12 Busam KJ, Chen YT, Old LJ, et al. Expression of melanA (MART1) in benign melanocytic nevi and primary cutaneous malignant melanoma. Am J Surg Pathol 1998;22:976-982.

13 Prieto VG, Woodruff JM. Expression of HMB45 antigen in spindle cell melanoma. J Cutan Pathol 1997;24: $580-581$.

14 Al-Batran SE, Rafiyan MR, Atmaca A, et al. Intratumoral T-cell infiltrates and MHC class I expression in patients with stage IV melanoma. Cancer Res 2005;65:3937-3941.

15 Hashimoto Y, Ito Y, Kato T, et al. Expression profiles of melanogenesis-related genes and proteins in acquired melanocytic nevus. J Cutan Pathol 2006;33:207-215.

16 Ruiter DJ, Brocker EB. Immunohistochemistry in the evaluation of melanocytic tumors. Semin Diagn Pathol 1993;10:76-91.

17 Smoller BR, McNutt NS, Hsu A. HMB-45 staining of dysplastic nevi. Support for a spectrum of progression toward melanoma. Am J Surg Pathol 1989;13:680-684.

18 Sun J, Morton Jr TH, Gown AM. Antibody HMB-45 identifies the cells of blue nevi. An immunohisto- chemical study on paraffin sections. Am J Surg Pathol 1990;14:748-751.

19 Wood WS, Tron VA. Analysis of HMB-45 immunoreactivity in common and cellular blue nevi. J Cutan Pathol 1991;18:261-263.

20 Rimm DL. What brown cannot do for you. Nat Biotechnol 2006;24:914-916.

21 Camp RL, Chung GG, Rimm DL. Automated subcellular localization and quantification of protein expression in tissue microarrays. Nat Med 2002;8:1323-1327. Epub 2002 Oct 21.

22 Kallioniemi OP, Wagner U, Kononen J, et al. Tissue microarray technology for high-throughput molecular profiling of cancer. Hum Mol Genet 2001;10:657-662.

23 Gown AM, Vogel AM, Hoak D, et al. Monoclonal antibodies specific for melanocytic tumors distinguish subpopulations of melanocytes. Am J Pathol 1986;123: 195-203.

24 Crotty KA, Scolyer RA, Li L, et al. Spitz naevus vs Spitzoid melanoma: when and how can they be distinguished? Pathology 2002;34:6-12.

25 Wechsler J, Bastuji-Garin S, Spatz A, et al. Reliability of the histopathologic diagnosis of malignant melanoma in childhood. Arch Dermatol 2002;138:625-628.

26 King MS, Porchia SJ, Hiatt KM. Differentiating Spitzoid melanomas from Spitz nevi through CD99 expression. J Cutan Pathol 2007;34:576-580.

27 McNutt NS, Urmacher C, Hakimian J, et al. Nevoid malignant melanoma: morphologic patterns and immunohistochemical reactivity. J Cutan Pathol 1995;22: 502-517. 